\title{
Synthesis of Anthracene-Bridged Dinuclear Phenoxyiminato Organotitanium Catalysts with Enhanced Activity, Thermal Stability and Comonomer Incorporation Ability toward Ethylene (Co)Polymerization
}

Shaofeng Liu, * Yanhong Xing, Quande Zheng, Yutong Jia, and Zhibo Li*

Key Laboratory of Biobased Polymer Materials, College of Polymer Science and Engineering, Qingdao University of Science and Technology, Qingdao, 266042, China 

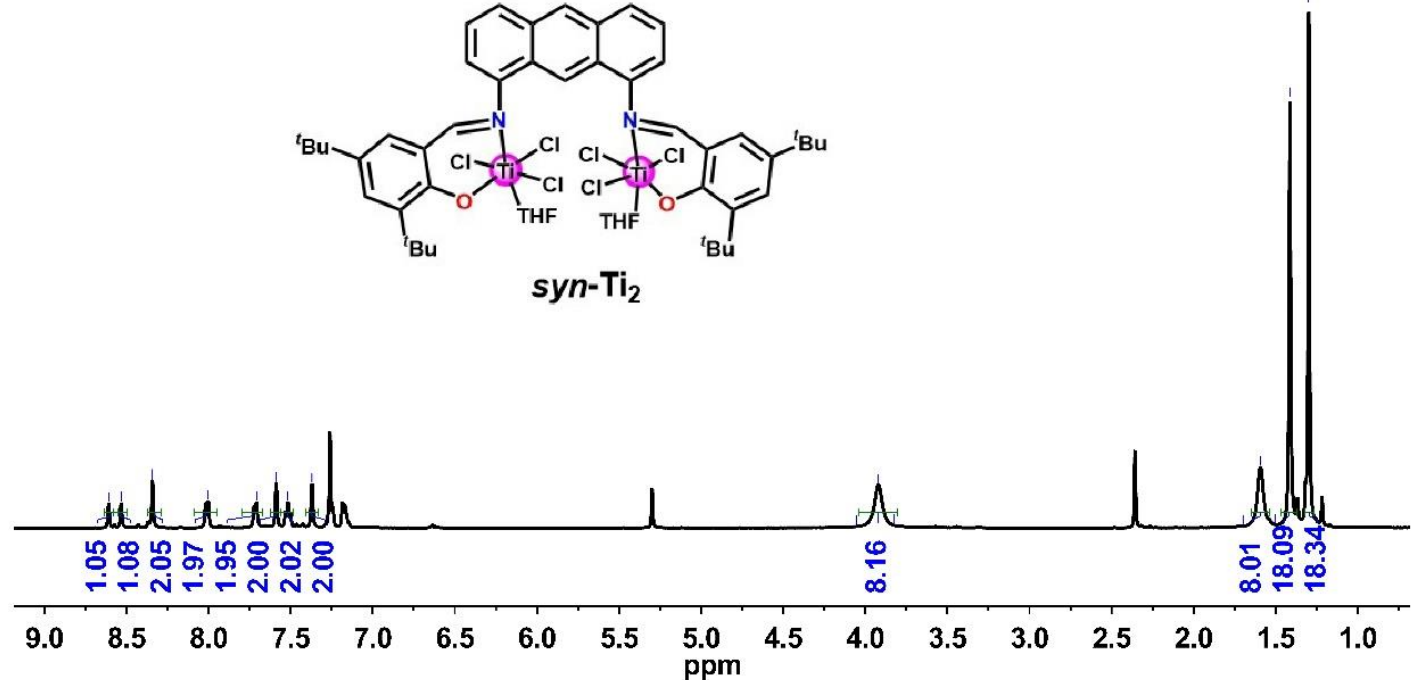

Figure S1. ${ }^{1} \mathrm{H}$ NMR spectrum of $\boldsymbol{s y n}-\mathbf{T i}_{2}$ in $\mathrm{CDCl}_{3}$.

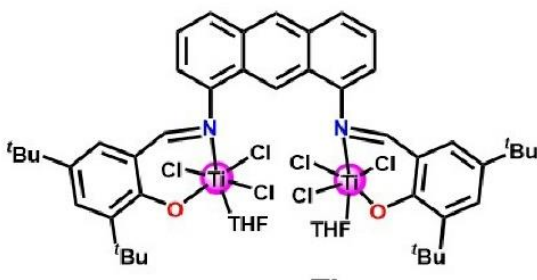

syn- $-\mathrm{Ti}_{2}$

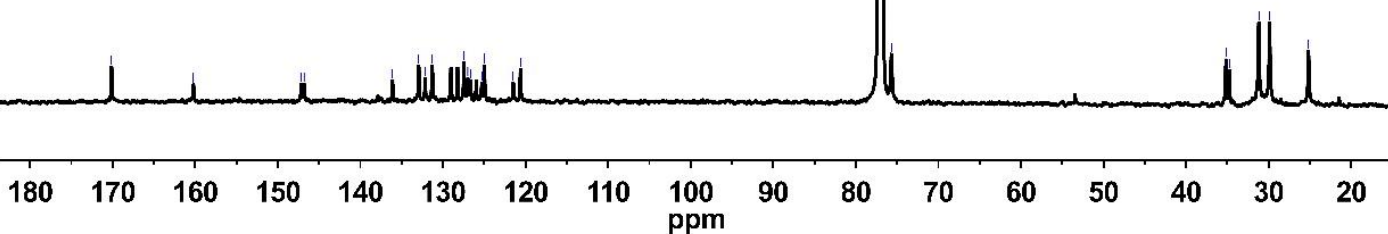

Figure S2. ${ }^{13} \mathrm{C}$ NMR spectrum of $\boldsymbol{s y n}$-Ti $\mathbf{i}_{2}$ in $\mathrm{CDCl}_{3}$. 


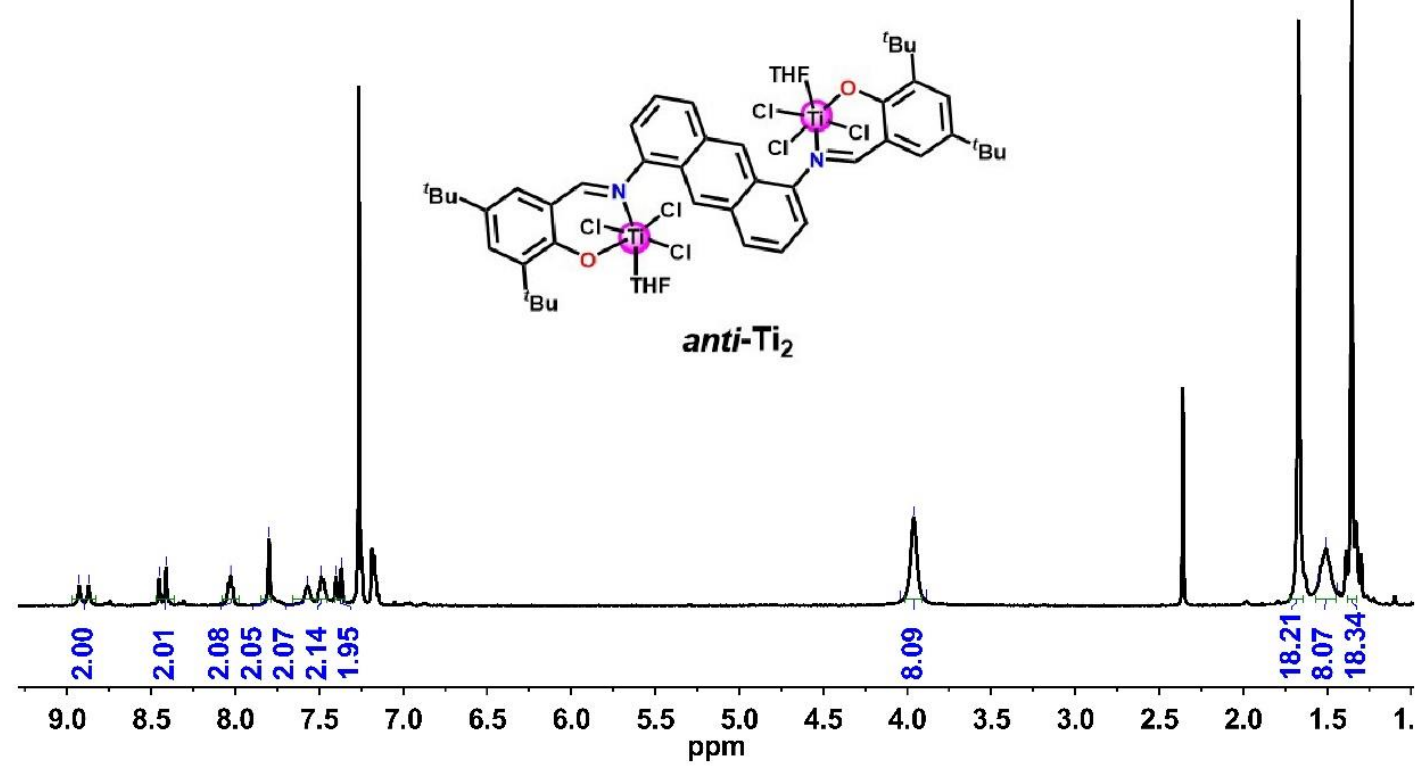

Figure S3. ${ }^{1} \mathrm{H}$ NMR spectrum of anti-Ti2 in $\mathrm{CDCl}_{3}$.

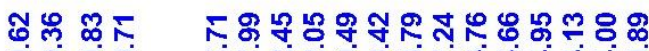

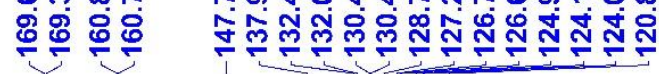

พั\&

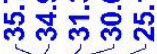
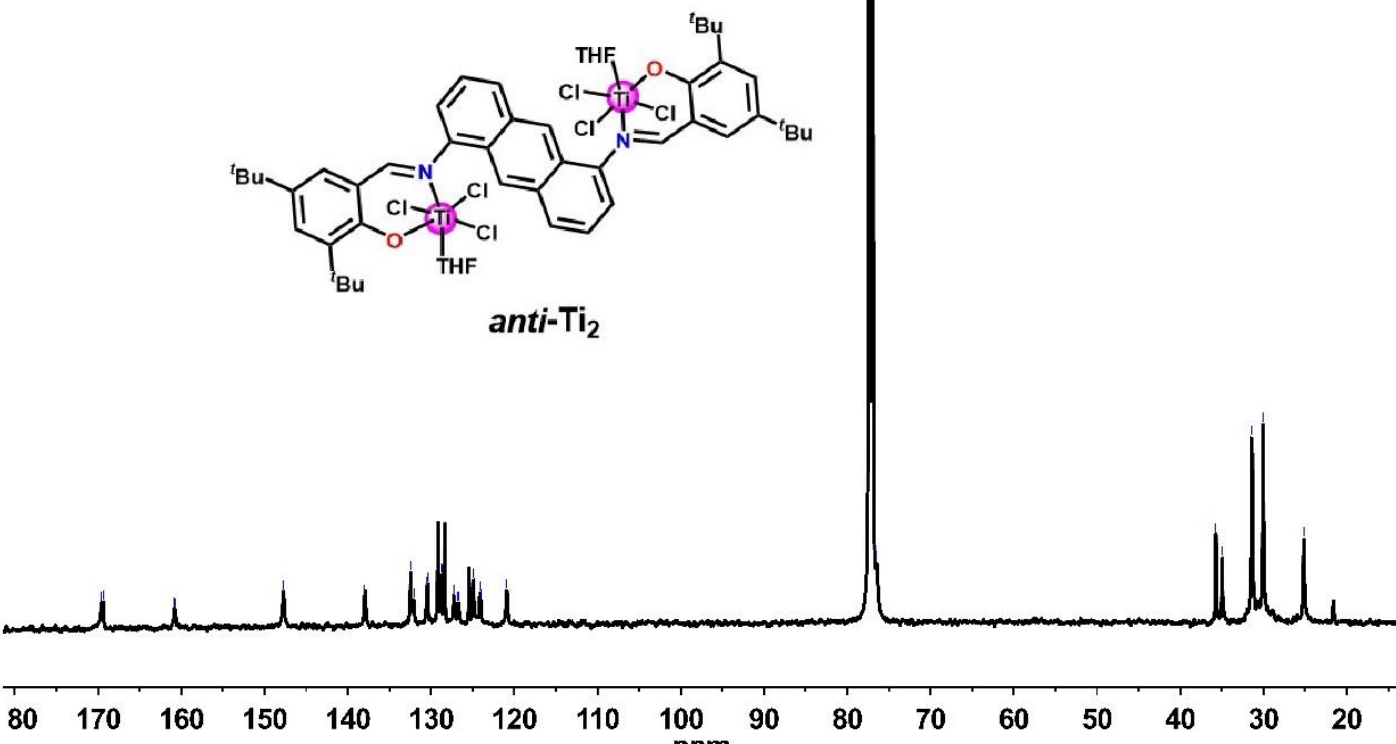

Figure S4. ${ }^{13} \mathrm{C}$ NMR spectrum of anti-Ti2 in $\mathrm{CDCl}_{3}$. 


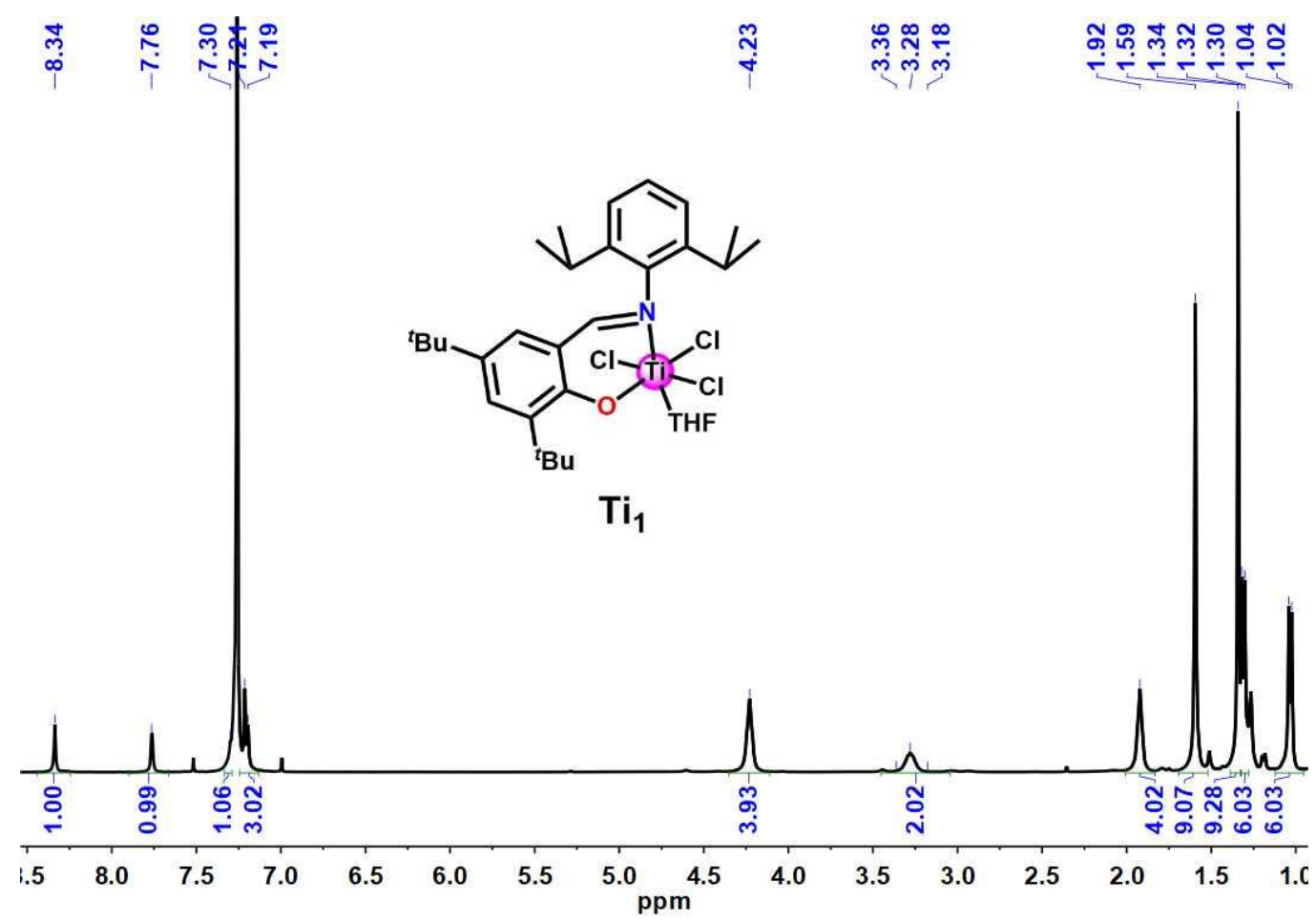

Figure S5. ${ }^{1} \mathrm{H}$ NMR spectrum of mononuclear $\mathbf{T i} 1$ in $\mathrm{CDCl}_{3}$.
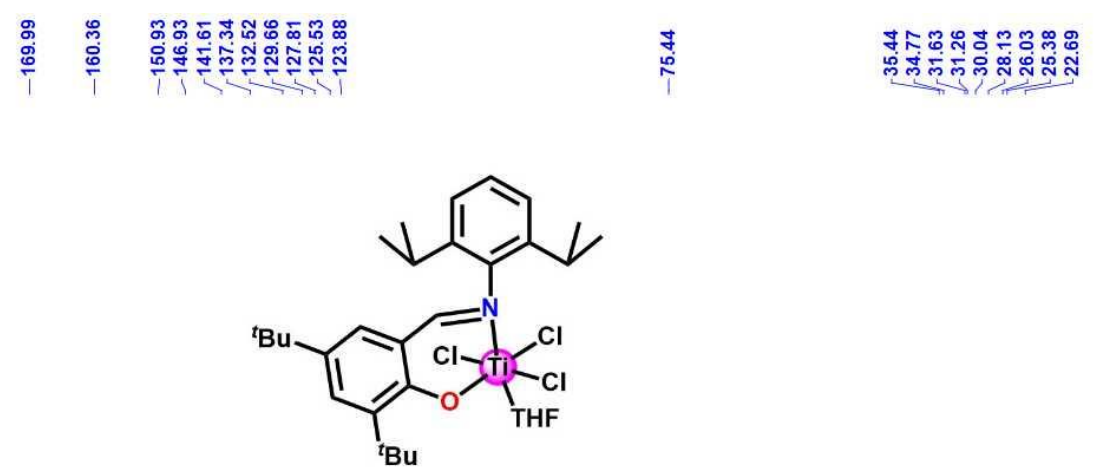

$\mathrm{Ti}_{1}$

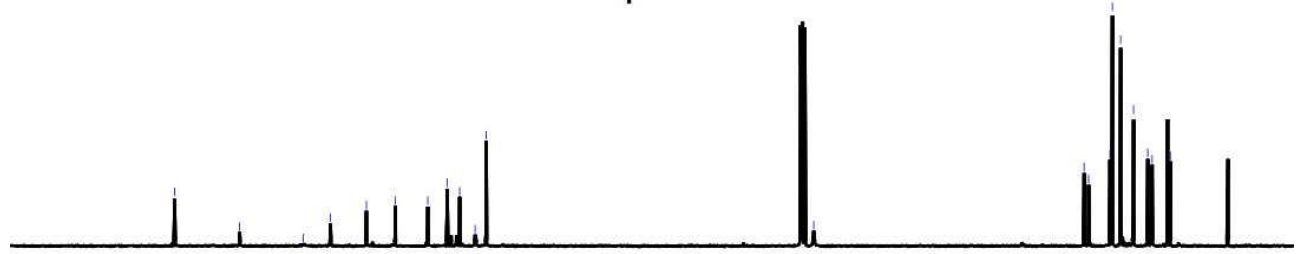

$\begin{array}{lllllllllllllllllll}190 & 180 & 170 & 160 & 150 & 140 & 130 & 120 & 110 & \begin{array}{l}100 \\ \mathrm{ppm}\end{array} & 90 & 80 & 70 & 60 & 50 & 40 & 30 & 20 & 10\end{array}$

Figure S6. ${ }^{13} \mathrm{C}$ NMR spectrum of mononuclear $\mathbf{T i} 1$ in $\mathrm{CDCl}_{3}$. 


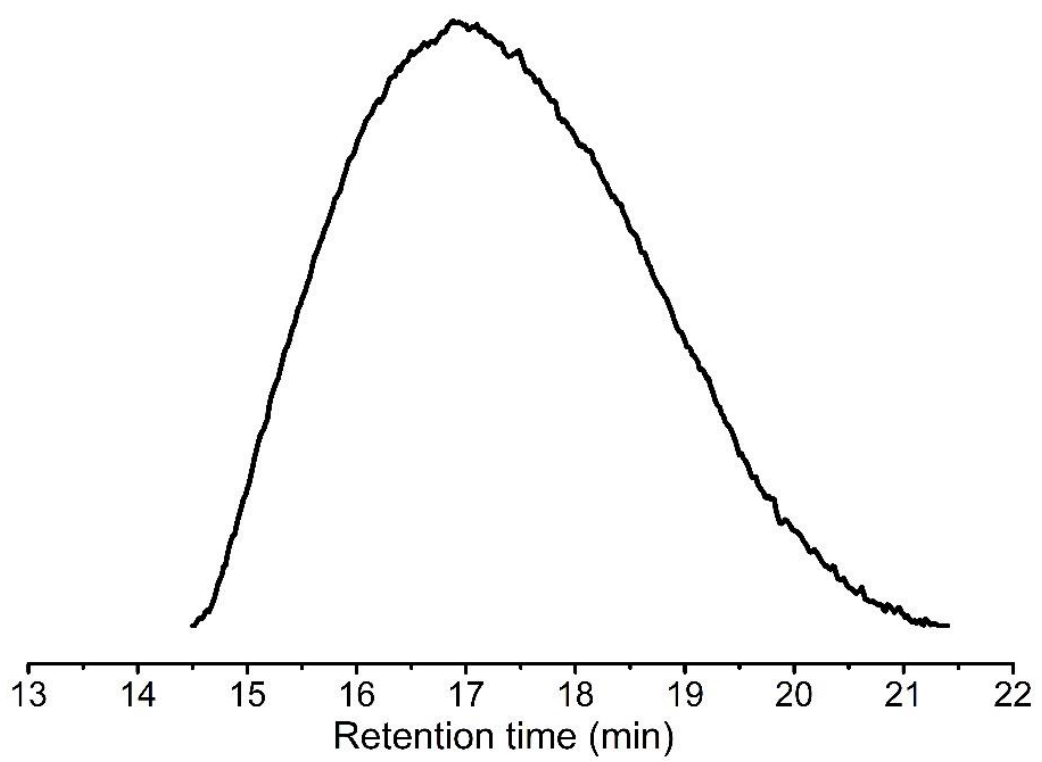

Figure S7. GPC trace of polymers obtained in Table 1 run 1.

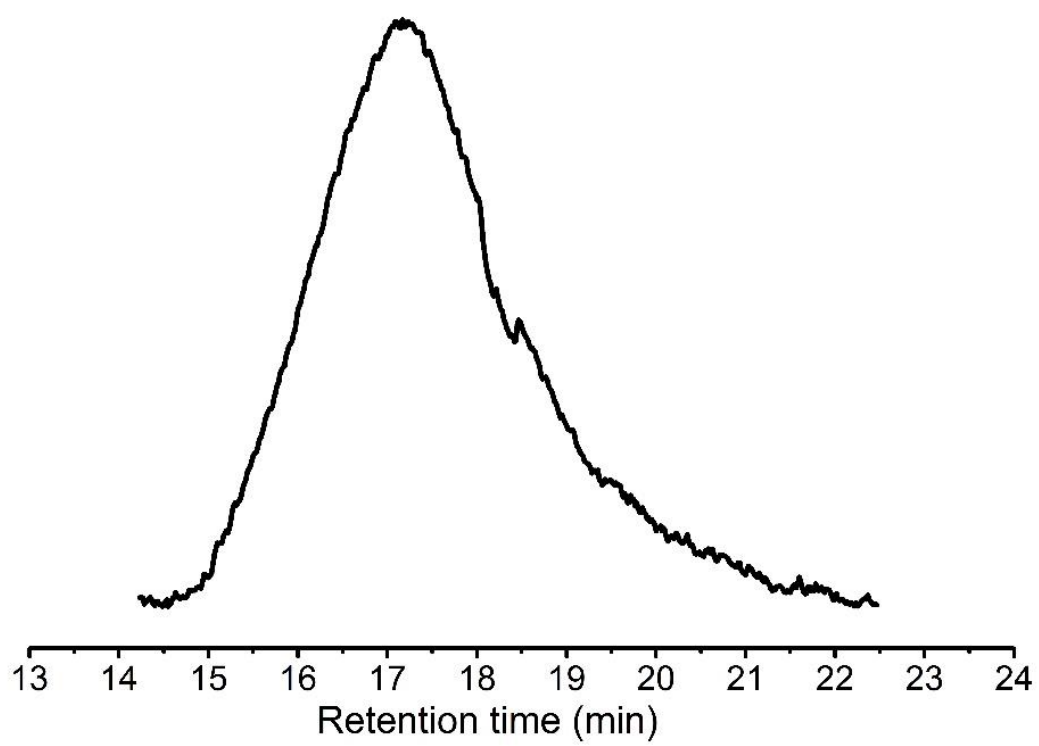

Figure S8. GPC trace of polymers obtained in Table 1 run 2. 


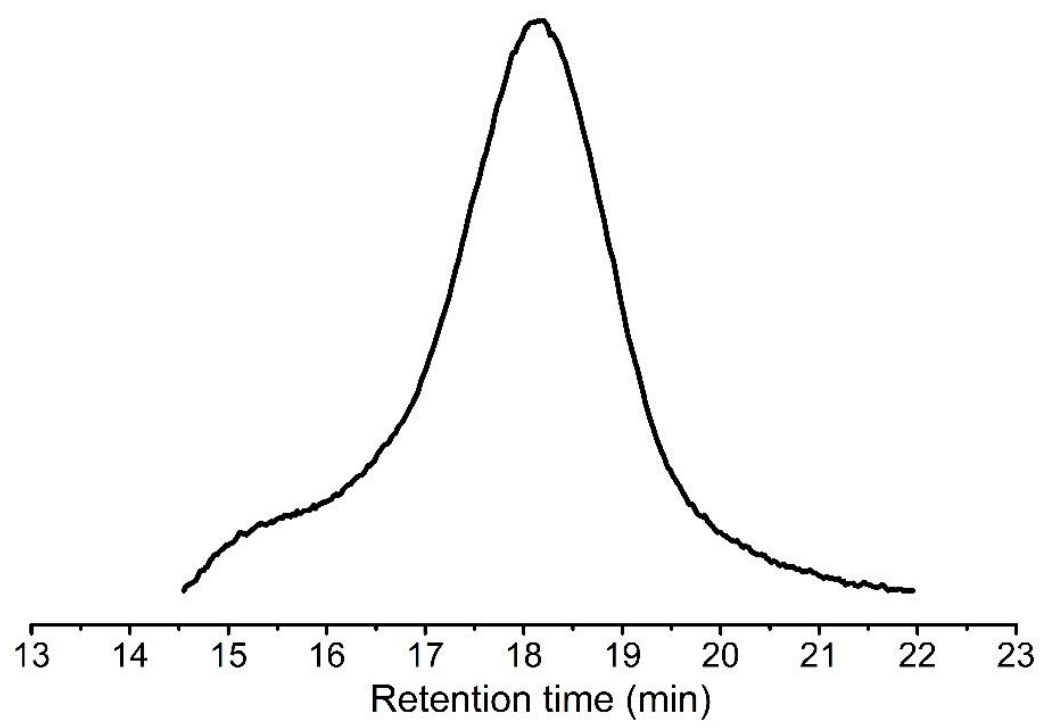

Figure S9. GPC trace of polymers obtained in Table 1 run 3.

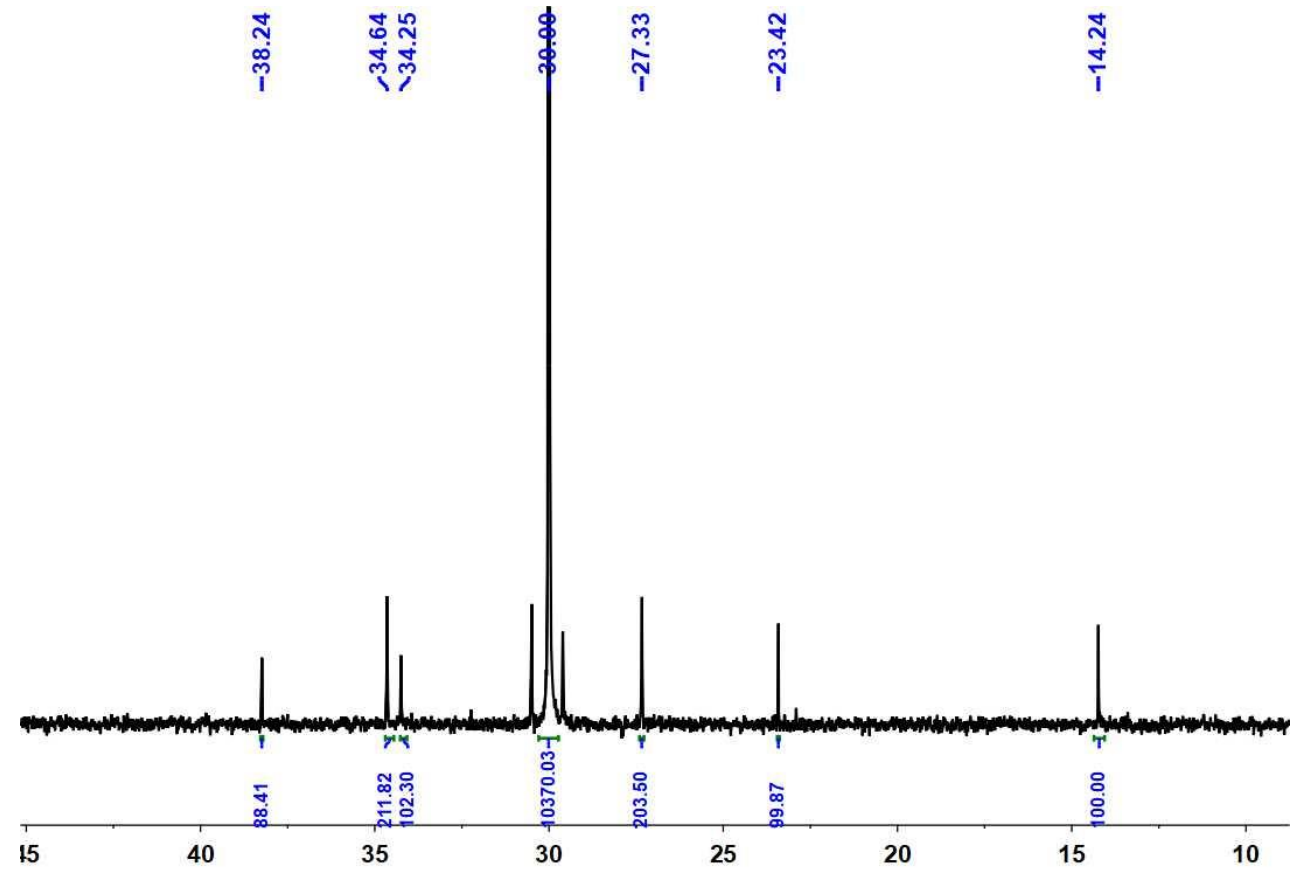

Figure S10. ${ }^{13} \mathrm{C}$ NMR spectrum of polymer obtained in Table 2 run 1 in $\mathrm{C}_{2} \mathrm{D}_{2} \mathrm{Cl}_{4}$ at $120{ }^{\circ} \mathrm{C}(1.8 \mathrm{~mol} \%$ of 1-hexene incorporation). 


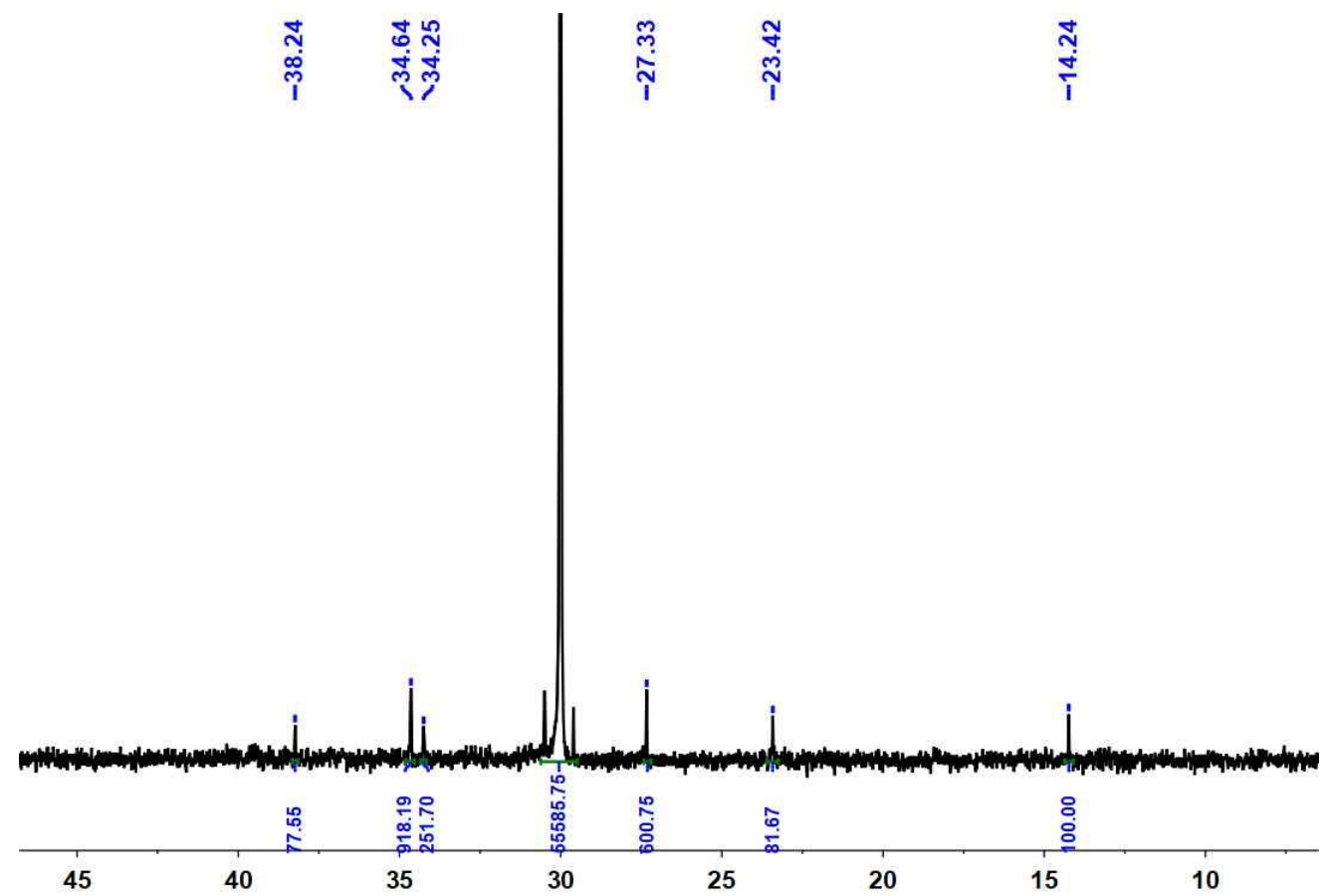

Figure S11. ${ }^{13} \mathrm{C}$ NMR spectrum of polymer obtained in Table 2 run 2 in $\mathrm{C}_{2} \mathrm{D}_{2} \mathrm{Cl}_{4}$ at $120{ }^{\circ} \mathrm{C}(0.3 \mathrm{~mol} \%$ of 1 -hexene incorporation). 
Table S1. Crystal data and structure refinement for $\mathbf{s y n}-\mathbf{T i} \mathbf{i}_{2} \cdot 2 \mathrm{CHCl}_{3}$.

\begin{tabular}{ll}
\hline Complex & syn-Ti2 $2 \mathrm{CHCl}_{3}$ \\
\hline Empirical formula & $\mathrm{C}_{52} \mathrm{H}_{64} \mathrm{Cl}_{6} \mathrm{~N}_{2} \mathrm{O}_{4} \mathrm{Ti}_{2} \cdot 2 \mathrm{CHCl}_{3}$ \\
Formula weight & 1257.39 \\
Temperature $/ \mathrm{K}$ & 100 \\
Crystal system & Triclinic \\
Space group & $\mathrm{P}-1$ \\
& $12.967(4)$, \\
$\mathrm{a} / \AA, \mathrm{b} / \AA, \mathrm{c} / \AA$ & $13.988(5)$, \\
& $20.389(3)$ \\
& $96.116(9)$, \\
$\alpha /{ }^{\circ}, \beta /{ }^{\circ}, \gamma /{ }^{\circ}$ & $103.434(9)$, \\
& $117.017(6)$ \\
Volume / $\AA^{3}$ & $3107.1(15)$ \\
$\mathrm{Z}$ & 2 \\
$\rho_{\text {calc }} /$ mg mm \\
$\mu$
\end{tabular}

